\title{
Structural Characteristics of Al-Co-Ni Decagonal Quasicrystals and Crystalline Approximants
}

\author{
Kenji Hiraga $^{1}$, Tetsu Ohsuna ${ }^{1}$, Wei Sun ${ }^{1}$ and Kazumasa Sugiyama ${ }^{2}$ \\ 'Institute for Materials Research, Tohoku University, Sendai 980-8577, Japan \\ ${ }^{2}$ Department of Earth and Planetary Sicience, Graduate School of Science, The University of Tokyo, Tokyo 113-0033, Japan
}

\begin{abstract}
The structural characteristics of several modulations of Al-Co-Ni decagonal quasicrystals and crystalline approximants, all of which are found in and around an Al-Co-Ni decagonal phase with a wide compositional range, are discussed systematically. The structures of a $\mathrm{Ni}$-rich basic structure and the other modulations are characterized as quasiperiodic arrangements of columnar clusters of atoms with decagonal sections of 3.2 and $2.0 \mathrm{~nm}$ in diameter, respectively. Various types of electron diffraction patterns including so-called superlattice reflections are interpreted by tiling features of the atomic clusters as well as ordered arrangements of the atomic clusters with pentagonal symmetry in the tilings. From the structure of a crystalline approximant (W-(AlCoNi) phase), precise models of the atomic clusters are proposed, and also the origin of diffuse reflections often observed in the $\mathrm{Al}-\mathrm{Co}-\mathrm{Ni}$ decagonal quasicrystals may be interpreted.
\end{abstract}

(Received August 8, 2001; Accepted September 25, 2001)

Keywords: quasicrystal, decagonal quasicrystal, decagonal phase, structure, transmission electron microscopy, high-angle annular detector dark-field scanning transmission electron microscopy, aluminum-cobalt-nickel

\section{Introduction}

The Al-Co-Ni decagonal phase has received much attention, because of the appearance of several modulations of decagonal quasicrystals, showing highly-ordered diffraction patterns or several different types of diffraction patterns with superlattice reflections, and some crystalline approximants. ${ }^{1-6)}$ Highly-ordered diffraction patterns, which can be interpreted from a pentagonal Penrose tiling, ${ }^{7)}$ with a period of $0.4 \mathrm{~nm}$ along the periodic axis have been observed in the $\mathrm{Ni}$-rich side. Therefore, this decagonal quasicrystal is called a Ni-rich basic structure. ${ }^{5)}$ On the other hand, decagonal quasicrystals with various types of diffraction patterns, most of which include so-called superlattice reflections, have been observed in the Co-rich side. In the diffraction patterns of these decagonal quasicrystals in the Co-rich side, diffuse reflections showing periodicity of $0.8 \mathrm{~nm}$ have been observed, and the intensity of the diffuse reflection enhances with increasing Co content. ${ }^{8)}$ That is to say, in the Al-Co-Ni decagonal phase, there exist a variety of polymorphism of decagonal quasicrystals, which have different structures on quasiperiodic planes perpendicular to the periodic axis and different periods along the periodic axis. In order to reveal the structures of these decagonal quasicrystals, many studies have been made by electron diffraction analysis, high-resolution transmission electron microscopy (HRTEM) and high-angle annular detector dark-field scanning transmission electron microscopy (HAADF-STEM). However, previous studies have been made on individual decagonal quasicrystals, and no systematic interpretation on the structures of all the Al-Co-Ni decagonal quasicrystals has been performed. Recently, precise structural analysis of a crystalline approximant, which is found in alloys around $\mathrm{Al}_{72.5} \mathrm{Co}_{20.5} \mathrm{Ni}_{7}$, annealed at $950^{\circ} \mathrm{C}$ for a long time, ${ }^{9)}$ has been performed by $\mathrm{X}$-ray diffraction analysis using a single crystal. ${ }^{10)}$ The structure of the crystalline approximant, which is called a W-(AlCoNi) crystal, enables us to discuss the structures of the Al-Co-Ni decagonal quasicrystals in detail. The purpose of the present work is to discuss systematically the structural characteristics of $\mathrm{Al}-\mathrm{Co}-\mathrm{Ni}$ decagonal quasicrystals on the basis of results of our previous studies on the modulations of the $\mathrm{Al}-\mathrm{Co}-\mathrm{Ni}$ decagonal quasicrystal, S1-type, ${ }^{11)}$ type-I, ${ }^{12)}$ type-II ${ }^{13)}$ and pentagonal superstructure, ${ }^{14)} \mathrm{Ni}$-rich ${ }^{15)}$ and $\mathrm{Co}^{- \text {rich }^{16)}}{ }^{16}$ basic structures and one-dimensional quasicrystal, ${ }^{17)}$ and two crystalline approximants. ${ }^{9,18)}$

\section{Experimental Procedures}

All Al-Co-Ni alloys presented in this paper are listed in

Table 1 Al-Co-Ni alloys used in the present paper, heat treatments and structures.

\begin{tabular}{lll}
\hline \multicolumn{1}{c}{ Alloys } & Heat treatments & Structures \\
\hline $\mathrm{Al}_{72} \mathrm{Co}_{8} \mathrm{Ni}_{20}$ & $900^{\circ} \mathrm{C}, 14 \mathrm{~h}$ & Ni-basic structure \\
$\mathrm{Al}_{70} \mathrm{Co}_{12} \mathrm{Ni}_{18}$ & $900^{\circ} \mathrm{C}, 48 \mathrm{~h}$ & S1-type superstructure \\
$\mathrm{Al}_{72.5} \mathrm{Co}_{11} \mathrm{Ni}_{16.5}$ & $900^{\circ} \mathrm{C}, 40 \mathrm{~h}$ & $\mathrm{~S} 1$-type superstructure \\
$\mathrm{Al}_{71} \mathrm{Co}_{14.5} \mathrm{Ni}_{14.5}$ & $1000^{\circ} \mathrm{C}, 65 \mathrm{~h}$ & Type-I superstructure \\
$\mathrm{Al}_{71.5} \mathrm{Co}_{16} \mathrm{Ni}_{12.5}$ & $900^{\circ} \mathrm{C}, 72 \mathrm{~h}$ & Type-II superstructure \\
$\mathrm{Al}_{71.5} \mathrm{Co}_{16} \mathrm{Ni}_{12.5}$ & $900^{\circ} \mathrm{C}, 120 \mathrm{~h}$ & Crystalline approximant \\
$\mathrm{Al}_{72.5} \mathrm{Co}_{17.5} \mathrm{Ni}_{10}$ & $900^{\circ} \mathrm{C}, 40 \mathrm{~h}$ & Co-rich basic structure \\
$\mathrm{Al}_{71} \mathrm{Co}_{19} \mathrm{Ni}_{10}$ & $1100^{\circ} \mathrm{C}, 11 \mathrm{~h}$ & One-dimensional quasicrystal \\
$\mathrm{Al}_{72.5} \mathrm{Co}_{20} \mathrm{Ni}_{7.5}$ & $900^{\circ} \mathrm{C}, 280 \mathrm{~h}$ & W-(AlCoNi) crystalline approximant \\
$\mathrm{Al}_{71.5} \mathrm{Co}_{25.5} \mathrm{Ni}_{3}$ & $1160^{\circ} \mathrm{C}, 3 \mathrm{~h}$ & Pentagonal superstructure \\
\hline
\end{tabular}



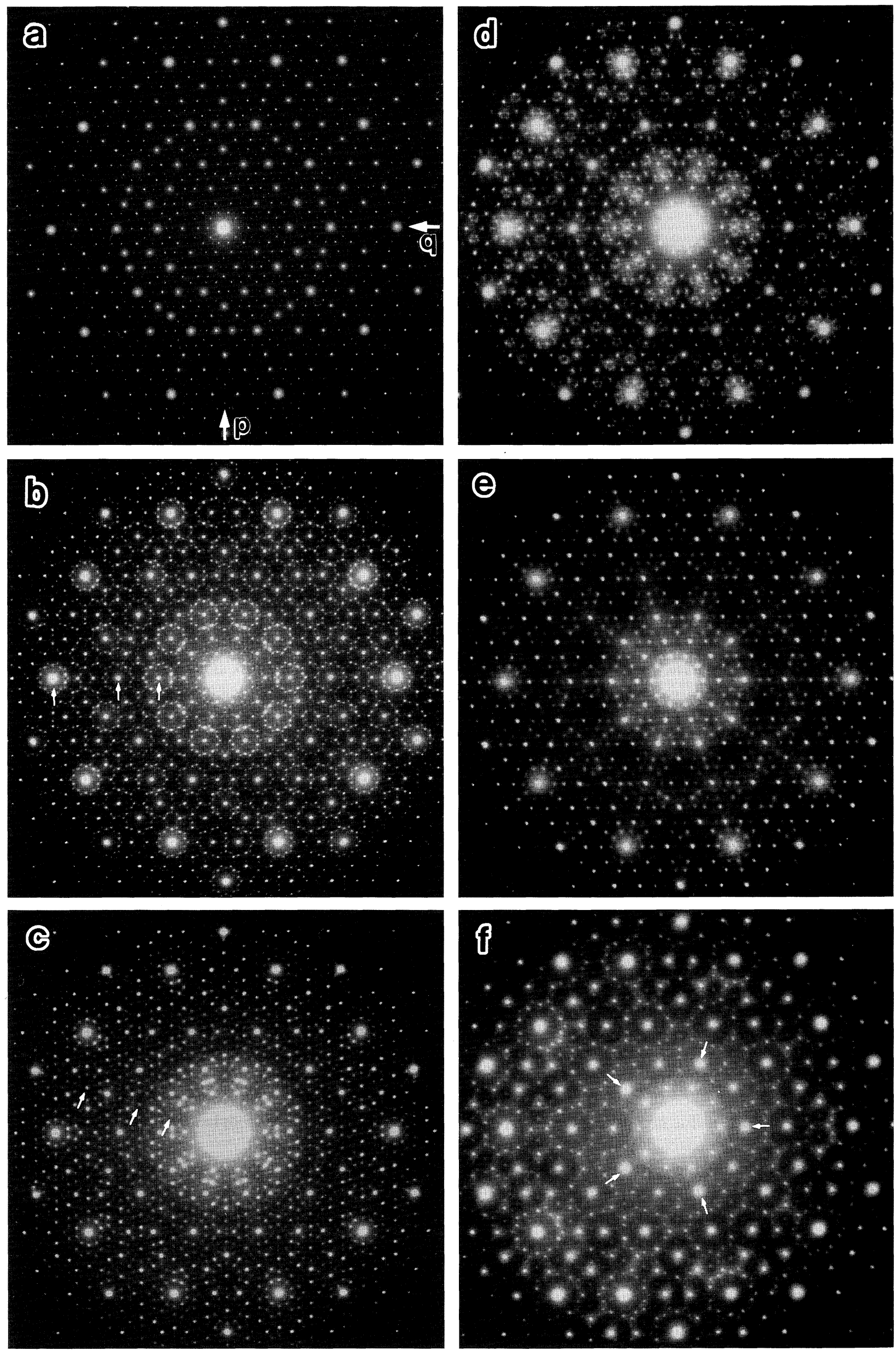


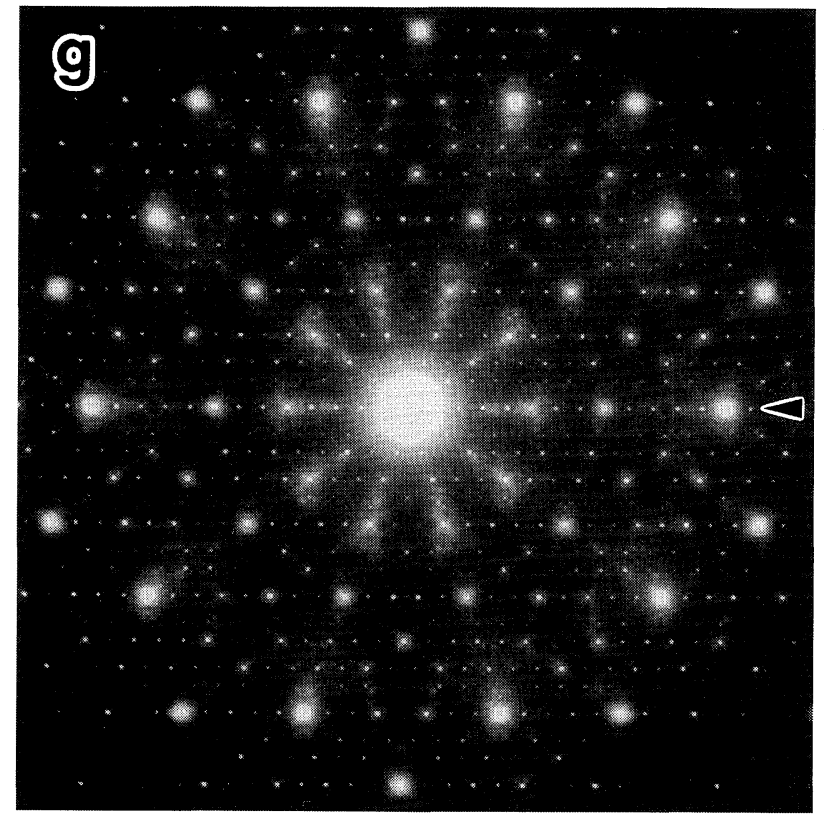

Fig. 1 Electron diffraction patterns of the Ni-rich basic structure (a), S1-type (b), type-I (c) and type-II (d) superstructures, Co-rich basic structure (e), pentagonal superstructure (f) and one-dimensional quasicrystal (g) of Al-Co-Ni decagonal quasicrystals, taken with the incident beam parallel to the periodic axis.

Table 1, with nominal compositions, heat treatments and structures. These alloys were prepared by melting highpurity (99.99\%) Al, Co and Ni metals in an arc furnace in an Ar atmosphere. The ingots were sealed in evacuated quartz tubes and annealed at various temperatures. Thin samples for electron microscopy were prepared by dispersing crushed materials on holey carbon films. HAADF-STEM observations were made on a $200 \mathrm{kV}$ transmission electron microscope (JEM-2010F) equipped with a scanning transmission mode (STEM). A beam probe with a half width of about $0.2 \mathrm{~nm}$ was scanned on the sample, and intensity of transmitted high-angle scattering beam was recorded with an annular detector of 60-160 mrad. The HAADF-STEM images presented in this paper are ones filtered by reconstructing using Fourier diffractograms of original images and apertures surrounding diffraction spots in the diffractograms, in order to reduce noise in the original images.

HRTEM observations were made on a $400 \mathrm{kV}$ transmission electron microscope (JEM-4000EX) with a resolution of $0.17 \mathrm{~nm}$.

\section{Electron Diffraction Patterns}

Figure 1 shows electron diffraction patterns of seven modulations of the Al-Co-Ni decagonal quasicrystal, taken with the incident beam parallel to the periodic axis. The intensity distributions of this type of diffraction patterns are attributed to two-dimensional structures projected along the periodic axis, and so result from the features of quasiperiodic lattices forming arrangements of atomic clusters and the structures of the atomic clusters located at their lattice points. That is to say, as a first approximation, a function showing a quasiperiodic structure is described by the convolution of two functions, viz., those showing a quasiperiodic lattice and a struc- ture of the atomic cluster at its lattice points, so that the intensity distribution of the diffraction pattern is determined by the product of Fourier transforms of those two functions.

Figures 1(a) and (e) are similar to diffraction patterns observed previously from many decagonal quasicrystals, ${ }^{19)}$ and all diffraction spots in these patterns can be interpreted by a standard pentagonal Penrose lattice. ${ }^{7)}$ Therefore, the decagonal quasicrystals showing Figs. 1(a) and (e) are called "Ni-rich and Co-rich basic structures", respectively. ${ }^{5)}$ On the other hand, the patterns of Figs. 1(b), (c), (d) and (f) include many extra spots, most of which are superlattice reflections. Therefore, the decagonal quasicrystals showing Figs. 1(b), (c) and (d) are called "S1-type, type-I and type-II superstructures", respectively. ${ }^{5)}$ It should be noted in Figs. 1(b) and (c) that intensity distributions of the superlattice reflections show $\tau^{2}$-scaling ( $\tau=1.618 \cdots$ is the golden ratio), as can be seen from intensity distributions around spots, indicated with small arrows in Figs. 1(b) and (c), with $\tau$-scaling. On the other hand, fundamental reflections in Figs. 1(a) and (e) have intensity distributions with $\tau$-scaling. This feature has been explained theoretically. ${ }^{20)}$

Also, one can see pentagonal symmetry in the intensity distribution of Fig. 1(f), as indicated by small arrows. The pentagonal symmetry results from the break down of Friedel's law by dynamical scattering in electron diffraction, and shows that this quasicrystal has non-central symmetry. This quasicrystal is called "fivefold or pentagonal superstructure", 5) because Fig. 1(f) includes superlattice reflections. In the diffraction pattern of Fig. 1(g), one can see periodic arrangements of diffraction spots along the one-direction indicated by an arrowhead. This feature clearly shows the existence of the one-dimensional quasicrystal. In Fig. 1(g), also, weak diffuse scattering around strong spots is observed. It should be noted that the intensity distribution of the diffuse scattering is similar to that of the superlattice reflections in the type-I superstructure (Fig. 1(c)). All the diffraction patterns of Fig. 1 are similar to those observed by Ritsch et al. ${ }^{5 \text { ) }}$

Figures 2 and 3 show diffraction patterns of four modulations, the Ni-rich basic structure, S1-type superstructure, Co-rich basic structure and pentagonal superstructure, taken with the incident beam parallel to the $p$ and $q$ directions in Fig. 1(a), as examples. One can see from Fig. 2 that intensities of diffuse streaks, indicated by arrowheads, showing $0.8 \mathrm{~nm}$ periodicity increase with increasing Co content, and finally become spots with maximum peaks at positions associated with the golden ratio in the pentagonal superstructure (Fig. 2(d)). That is to say, the Al-Co-Ni decagonal quasicrystals continuouly change from 0.4 to $0.8 \mathrm{~nm}$ periodicity as increasing Co content. This tendency has been observed as the common one in all the modulations of the Al-Co-Ni decagonal quasicrystals.

On the other hand, Bragg spot lines, showing $0.4 \mathrm{~nm}$ periodicity, indicated by a white arrow in Fig. 2(b), disappear in only the pattern (Fig. 2(a)) of the Ni-rich basic decagonal quasicrystal, although they appear in the pattern of Fig. 3(a). This observation shows that only the Ni-rich basic structure has the $c$-glide ( $c$ is the periodic axis) plane or a $10_{5}$ screw axis along the $c$-axis, in regard to the structures of $0.4 \mathrm{~nm}$ periodicity. This feature can be understood from the difference in the structures of columnar clusters of atoms forming the 

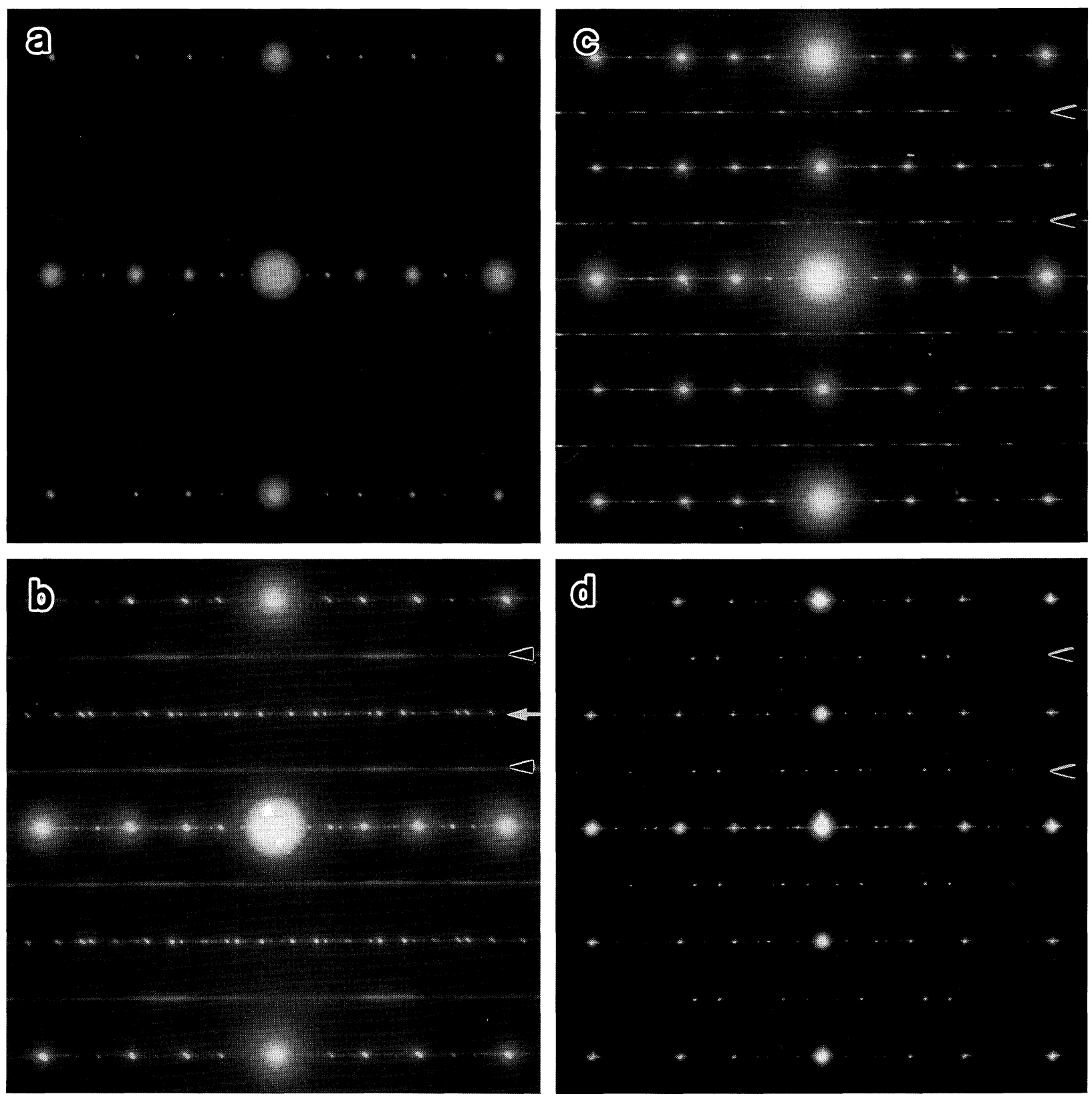

Fig. 2 Electron diffraction patterns of the Ni-rich basic structure (a), S1-type superstructure (b), the Co-rich basic structure (c) and pentagonal superstructure (d) of Al-Co-Ni decagonal quasicrystals, taken with the incident beam parallel to the $p$ direction in Fig. $1(\mathrm{a})$.

structures of the Ni-rich basic structure and the other modulations, as will be mentioned later.

\section{Arrangements of Atomic Clusters}

Columnar clusters of atoms with two different sizes of decagonal sections of $3.2 \mathrm{~nm}$ and $2.0 \mathrm{~nm}$ in diameter have been found in the modulations of Al-Co-Ni decagonal quasicrystals. These columnar clusters are hereafter called $3.2 \mathrm{~nm}$ and $2.0 \mathrm{~nm}$ clusters, respectively. The structures of six modulations, the S1-type, type-I and type-II superstructures, Co-rich basic structure, pentagonal superstructure and one-dimensional quasicrystal, are described as aperiodic arrangements of the $2.0 \mathrm{~nm}$ clusters, whereas the Ni-rich basic structure is formed by the $3.2 \mathrm{~nm}$ clusters. In Fig. 4, HAADF-STEM images of four modulations, the S1-type and
type-I superstructure, Co-rich basic structure and pentagonal superstructure, are shown as examples. The HAADF-STEM images were formed during scanning of the incident beam, so the sample drift during scanning produced the local deformations of image contrasts. Although there are local deformations in Fig. 4, one can easily notice peculiar contrasts consisting of small pentagonal arrangements of bright dots in all the images, and one can see that the pentagonal contrasts are arranged by aperiodic lattices with a bond length of $2.0 \mathrm{~nm}$, as indicated by lines. The pentagonal contrast is surrounded by twofold decagonal arrangements of bright dots in the $2.0 \mathrm{~nm}$ cluster, as indicated by a circle in Fig. 4(a). Detailed contrast distributions in the $2.0 \mathrm{~nm}$ clusters in HAADF-STEM images will be mentioned later.

One can notice that the $2.0 \mathrm{~nm}$ clusters are connected with each other with an interval of $2.0 \mathrm{~nm}$ and form aperiodic lat- 

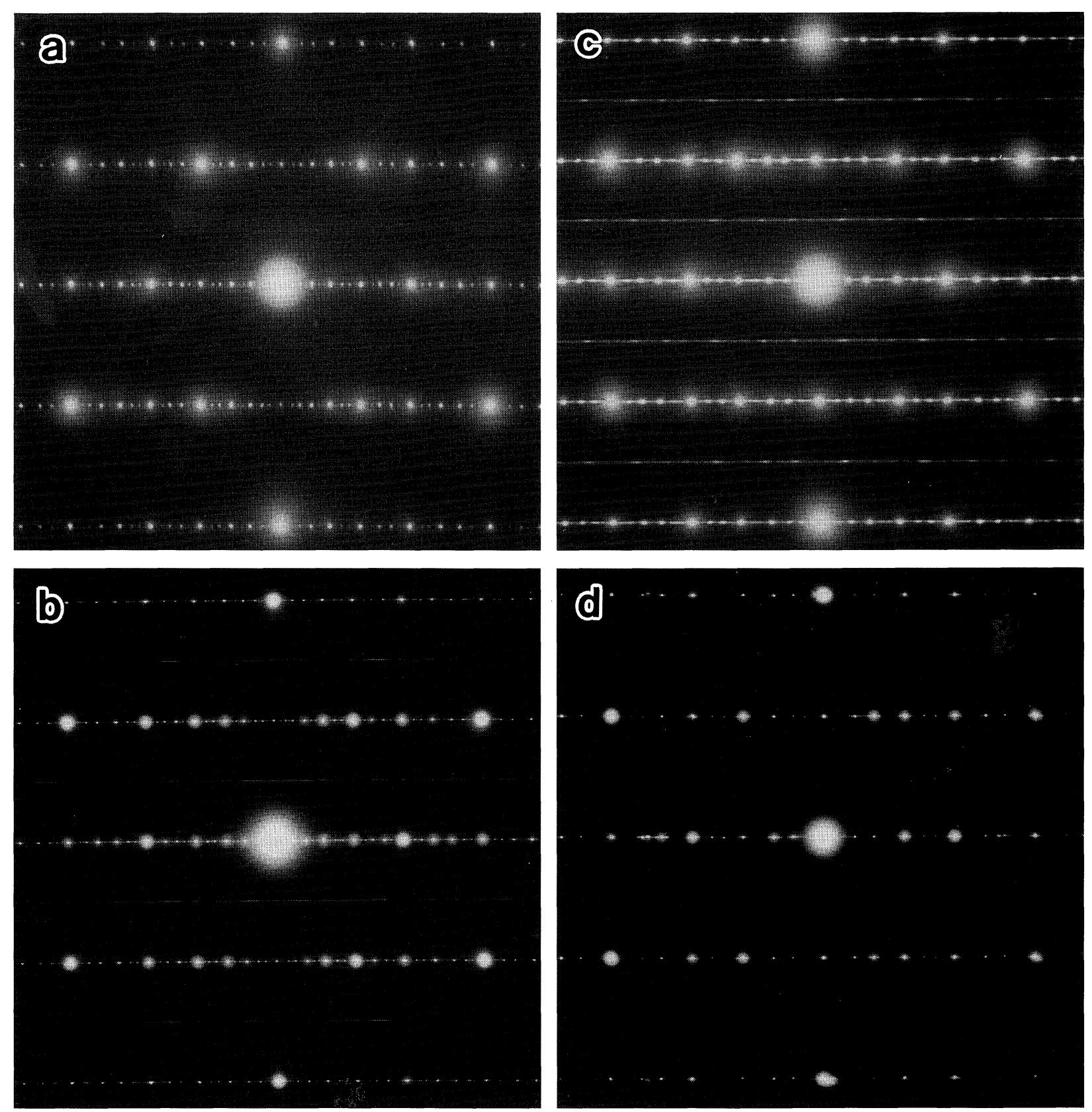

Fig. 3 Electron diffraction patterns of the Ni-rich basic structure (a), S1-type superstructure (b), the Co-rich basic structure (c) and pentagonal superstructure (d) of Al-Co-Ni decagonal quasicrystals, taken with the incident beam parallel to the $q$ direction in Fig. $1(\mathrm{a})$.

tices, as shown by lines in Fig. 4. Also, one can notice the existence of two types of pentagonal contrasts with two different orientations in Figs. 4(a) and (b), whereas all the pentagonal contrasts in Figs. 4(c) and (d) have the same orientation. The aperiodic lattices, formed by connecting the pentagonal contrasts, viz., the centers of the $2.0 \mathrm{~nm}$ clusters, in observed HAADF-STEM images, are shown in Fig. 5 for six modulations, viz., the S1-type (a), type-I (b) and type-II (c) superstructures, Co-rich basic structure (d), fivefold superstructure (e) and one-dimensional quasicrystal (f). In Fig. 5, two types of the clusters with different orientations of pentagonal symmetry are drawn by open and closed circles, respectively. It can be seen from Figs. 5(d) and (e) that the structures of the Co-rich basic structure and pentagonal superstructure are characterized as pentagonal and rhombic quasiperiodic arrangements of the $2.0 \mathrm{~nm}$ clusters with the same orientations, respectively. ${ }^{14,16)}$ Thus, both the structures have pentagonal symmetry, which results from the symmetry of the $2.0 \mathrm{~nm}$ clusters. That is to say, it can be concluded that the Co-rich basic structure and pentagonal superstructure can be characterized as pentagonal quasicrystals with pentagonal and rhombic quasiperiodic lattices, respectively,

On the other hand, the structures of the S1-type, type-I, type-II and one-dimensional quasicrystals are formed by two types of the $2.0 \mathrm{~nm}$ clusters with different orientations of pentagonal symmetry. The lattices in Figs. 5(a) and (b) can be characterized as pentagonal and rhombic quasiperiodic ones, respectively, because a deformed octagon existing mainly in Fig. 5(a) can be divided into two pentagons and one thin rhombus, and a hexagon in Fig. 5(b) into fat and thin rhom- 

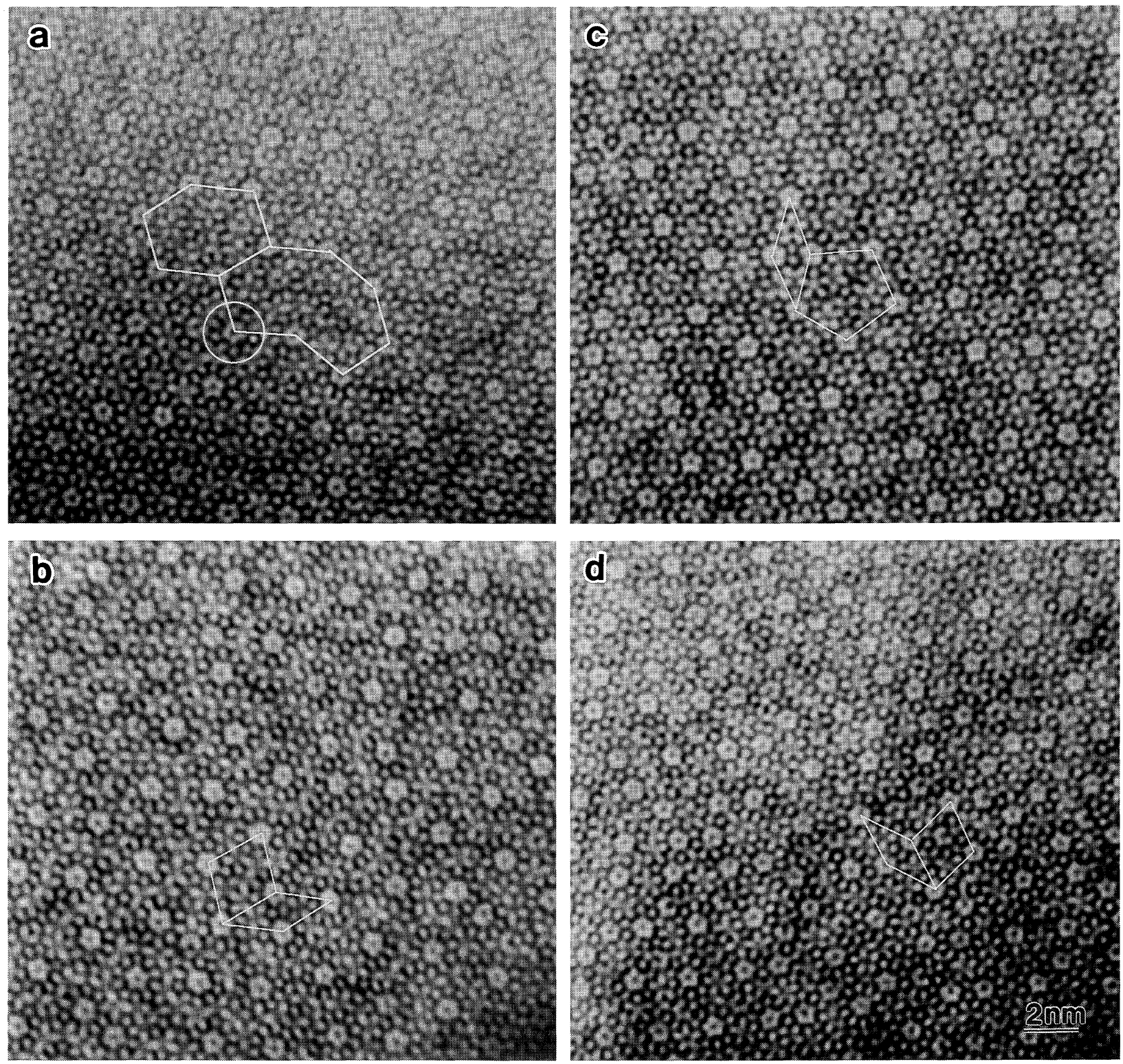

Fig. 4 HAADF-STEM images of the S1-type superstructure (a), type-I superstructure (b) the Co-rich basic structure (c) and pentagonal superstructure (d) of Al-Co-Ni decagonal quasicrystals, taken with the incident beam parallel to the periodic axis.

buses. In the arrangements of the two types of clusters in Figs. 5 (a) and (b), one can see the definite order that two clusters connected with a bond are always different types of clusters, though this order is broken at pentagonal tiles in Fig. 5(b), as indicated by dotted lines. The pentagonal and rhombic lattices with this ordered arrangement have been theoretically interpreted from the projections of $\mathrm{CsCl}$-type and $\mathrm{NaCl}$-type hypercubic lattices, respectively. ${ }^{21,22)} \mathrm{A}$ fundamental lattice of the $\mathrm{CsCl}$-type ordered decagonal quasicrystal can be divided into two sublattices of rhombic lattice with a bond length of $\tau a$ ( $a$ is a bond length of the fundamental lattice) ${ }^{21)}$

As for the type-II superstructure, its structure is characterized as a mixed state of the S1-type and type-I superstructures, namely pentagonal and rhombic quasiperiodic lattices. ${ }^{14)}$ However, one can see locally ordered arrangements of the clusters, as indicated by solid lines in Fig. 5(c). The local ordered arrangement of two types of clusters produces superlattice reflections in Fig. 1(d). On the other hand, in Fig. 5(f), one can see a heterogeneous distribution of open and closed circles, and a mixed state of pentagonal and rhombic lattices. However, in Fig. 5(f), lattice planes with high density of clusters along the one direction are periodically arranged, as indicated by arrows. That is to say, the structure of the one-dimensional quasicrystal has no perfect periodic structure along the one direction, but it is formed by the periodic array of lattice planes with high density of the clusters along the one direction. ${ }^{18)} \mathrm{A}$ defectively ordered arrangement of two types of the clusters in Fig. 5(f) can be considered to produce weak diffuse reflection around strong spots in Fig. $1(\mathrm{~g})$.

From the lattices in Fig. 5, one can see the tendency that the density of clusters decreases with increasing Ni content, and finally the S1-type superstructure with the highest $\mathrm{Ni}$ content in the modulations of Fig. 5(a) has no thin rhombic lattices.

In the Ni-rich basic structure with higher $\mathrm{Ni}$ content than the S1-type superstructure, the $3.2 \mathrm{~nm}$ cluster becomes a structural unit, and the clusters are arranged with intervals of $3.2 \mathrm{~nm}$ and $2.0 \mathrm{~nm}$, as can be seen from Fig. 6. By view- 

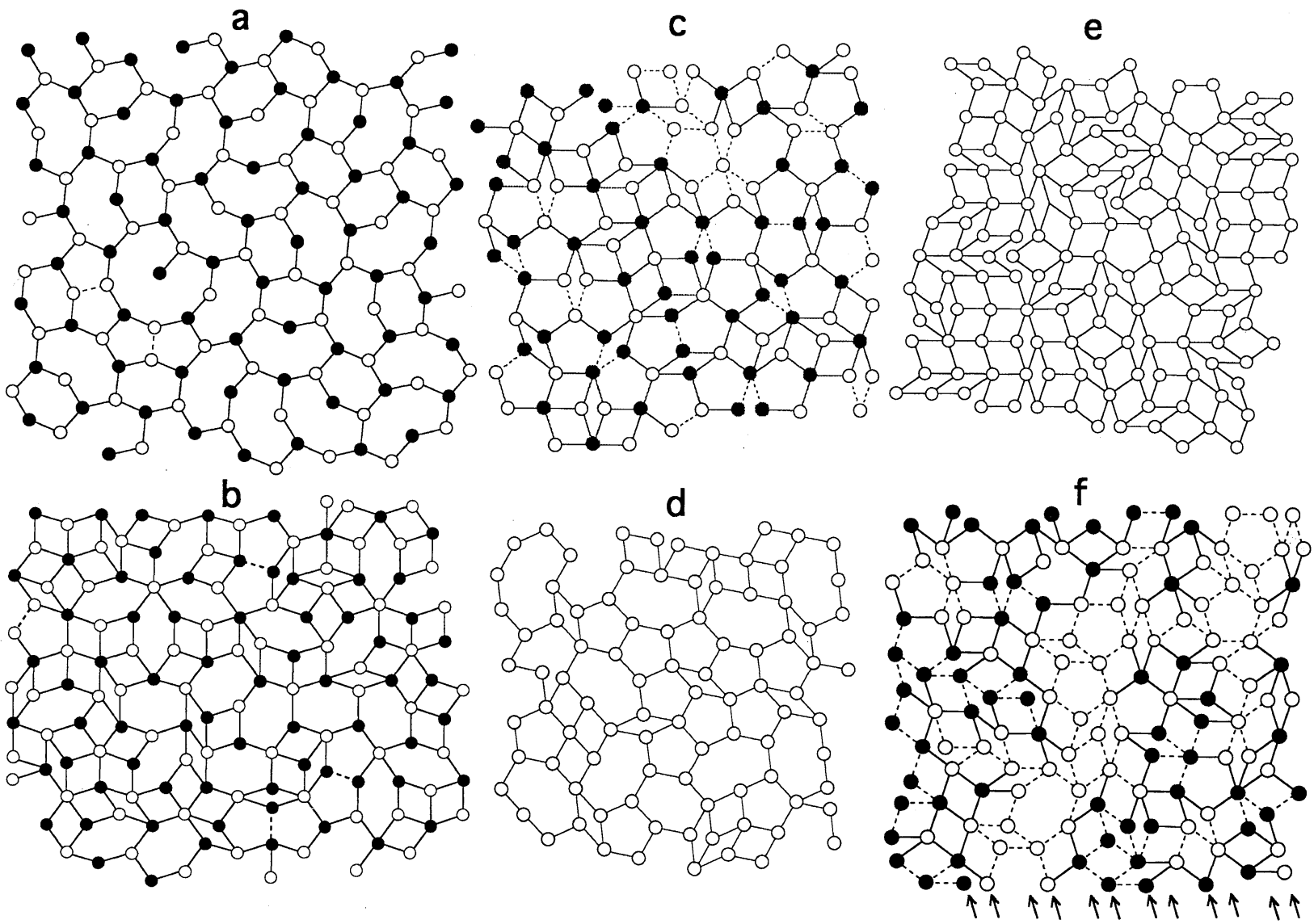

Fig. 5 Quasiperiodic lattices of the S1-type (a), type-I (b) and type-II (c) superstructures, Co-rich basic structure (d), pentagonal superstructure (e) and one-dimensional quasicrystal (f), obtained from HAADF-STEM observations. Open and closed circles indicate two types of clusters with different orientations of pentagonal symmetry. Solid and dotted lines show bonds binding different types and the same types of clusters, respectively.

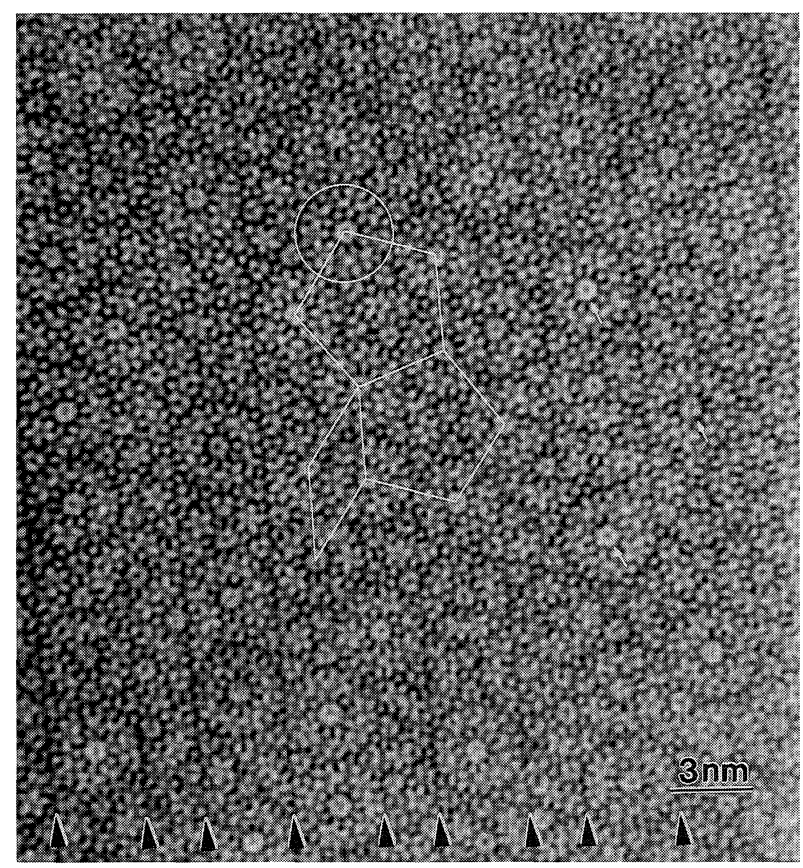

Fig. 6 HAADF-STEM image of the Ni-rich basic structure of Al-Co-Ni decagonal quasicrystal, taken with the incident beam parallel to the periodic axis. ing obliquely Fig. 6, taking attention of dark lines indicated with arrowheads, one can notice a pentagonal quasiperiodic lattice of the $3.2 \mathrm{~nm}$ clusters indicated by a circle, as shown by lines. It should be noted here that no definite contrasts are observed at the centers of the $3.2 \mathrm{~nm}$ clusters, and that a variety of contrast distributions with pentagonal, ring and triangle shapes, which are indicated with small arrows in Fig. 6, are observed at the centers of the clusters. This feature of the central contrasts in the $3.2 \mathrm{~nm}$ clusters has been also found in HAADF-STEM images of highly-ordered Al-Cu- $\mathrm{Rh}^{23)}$ and $\mathrm{Al}-\mathrm{Fe}-\mathrm{Ni}^{24)}$ decagonal quasicrystals with $0.4 \mathrm{~nm}$ periodicity. Also, the variety of arrangements of the atoms around the centers of atomic clusters have been found in some crystalline approximants. ${ }^{25,26)}$ The contrast distributions at the centers of the $3.2 \mathrm{~nm}$ in Fig. 7(a) can be assumed to have a decagonal distribution of bright contrasts on average, because the diffraction patterns of the Ni-basic structure (Figs. 2(a) and 3(a)) shows the existence of the $c$-glide ( $c$ is the periodic axis) plane or a $10_{5}$ screw axis along the $c$-axis. On the other hand, the pentagonal symmetry of the $2.0 \mathrm{~nm}$ clusters observed HAADF-STEM images (Fig. 4) clearly shows pentagonal symmetry with no $c$-glide plane, as shown in the diffraction patterns of Figs. 2 and 3.

\section{Two types of Atomic Clusters}

Figures 7 shows HRTEM images, which clearly show con- 

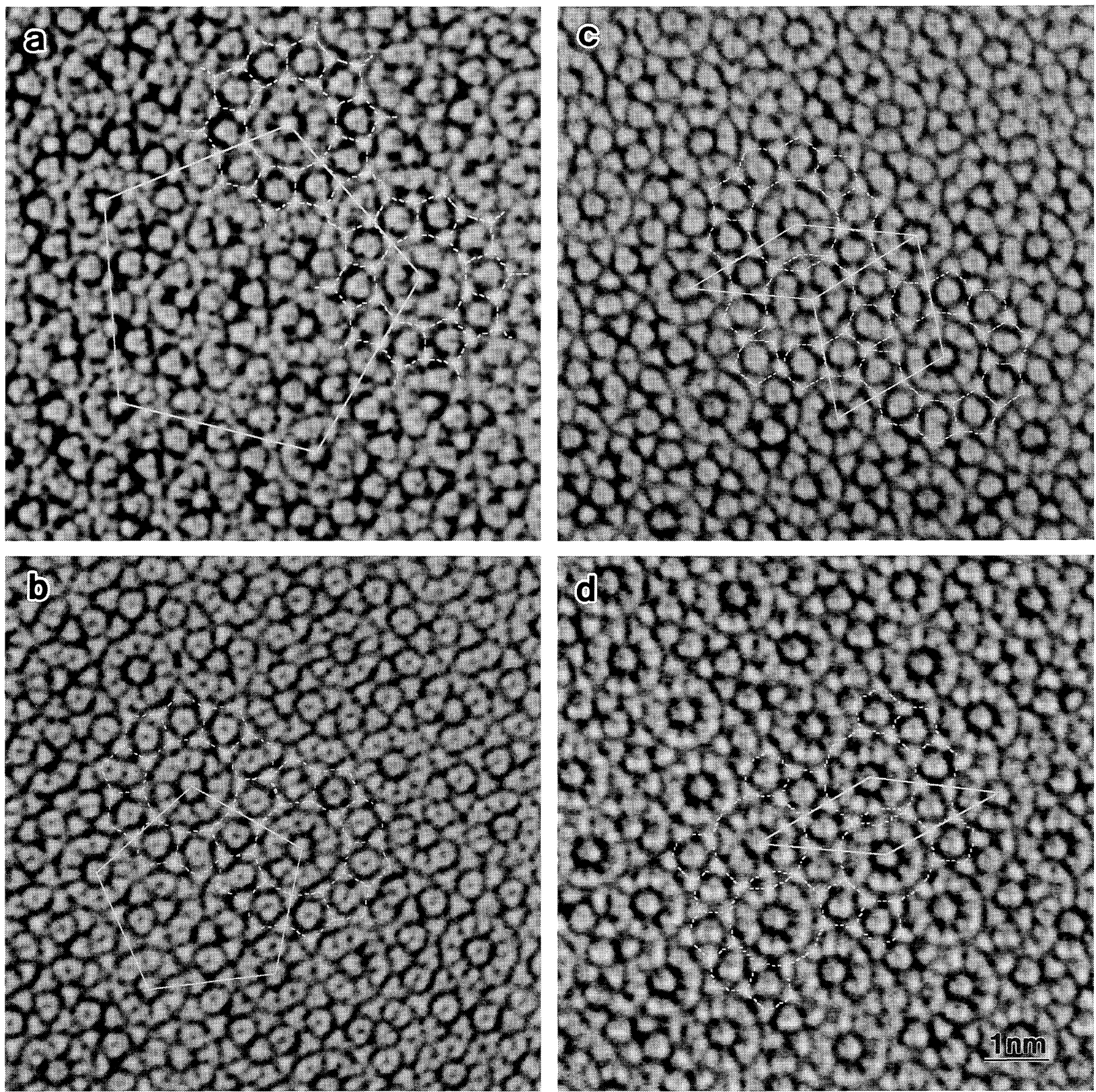

Fig. 7 HRTEM images showing atomic clusters, in the Ni-rich basic structure (a), S1-type superstructure (b), type-I superstructure (c) of Al-Co-Ni decagonal quasicrystals, taken with the incident beam parallel to the periodic axis, and in the $\mathrm{W}$-(AlCoNi) crystalline phase (d) taken with the incident beam parallel to the $b$-axis. Contrast distributions in the clusters are characterized as pentagonal and decagonal frames of dotted lines. Solid lines show lattices formed by connecting the centers of the atomic clusters.

trast distributions of the $3.2 \mathrm{~nm}$ and $2.0 \mathrm{~nm}$ clusters in the $\mathrm{Ni}$-rich basic structure, S1-type and type-I superstructure and W-(AlCoNi) crystalline approximant. In Fig. 7, one can see special image contrasts consisting of ring contrasts surrounding a wheel-like contrast. These contrast features can be characterized by a frame of pentagons surrounding a decagon, as shown by dotted lines in Fig. 7. The ring contrasts and wheel-like contrasts are located in the pentagonal and decagonal frames, respectively. The pentagonal and decagonal frames with a bond length of $0.47 \mathrm{~nm}$ and decagons of 2.0 and $3.2 \mathrm{~nm}$ in diameter can be described in a rhombic Penrose lattice of a bond length of $0.25 \mathrm{~nm}$, as shown in Fig. 8 . The structure of the Ni-rich basic decagonal quasicrystal is formed by edge-sharing and interpenetrating linkages of the $3.2 \mathrm{~nm}$ clusters, as shown in Fig. 9. The edge-sharing link- age of the $3.2 \mathrm{~nm}$ clusters can be clearly seen in Fig. 7(a). On the other hand, the interpenetrating linkage of Fig. 9(b) has a distance of $3.2 / \tau=2.0 \mathrm{~nm}$, which corresponds to a short diagonal of a thin rhombus with a bond length of $3.2 \mathrm{~nm}$.

The other modulations of the Al-Co-Ni decagonal quasicrystals have structures formed by aperiodic arrangements of the $2.0 \mathrm{~nm}$ clusters, which are connected with each other by three linkages of Fig. 10. The edge-sharing linkage of the $2.0 \mathrm{~nm}$ clusters makes bonds of a $2.0 \mathrm{~nm}$ length, and the interpenetrating linkage with a distance of $2.0 / \tau \fallingdotseq 1.2 \mathrm{~nm}$ correspond to a short diagonal of the thin rhombus in Fig. 5. On the other hand, a short diagonal of a fat rhombus is about $2.35 \mathrm{~nm}$, and in this linkage, the deformation of pentagonal frames occurs, as shown in Fig. 10(c). Those linkages of the $2.0 \mathrm{~nm}$ clusters and the deformation of the pentagonal frames can be 

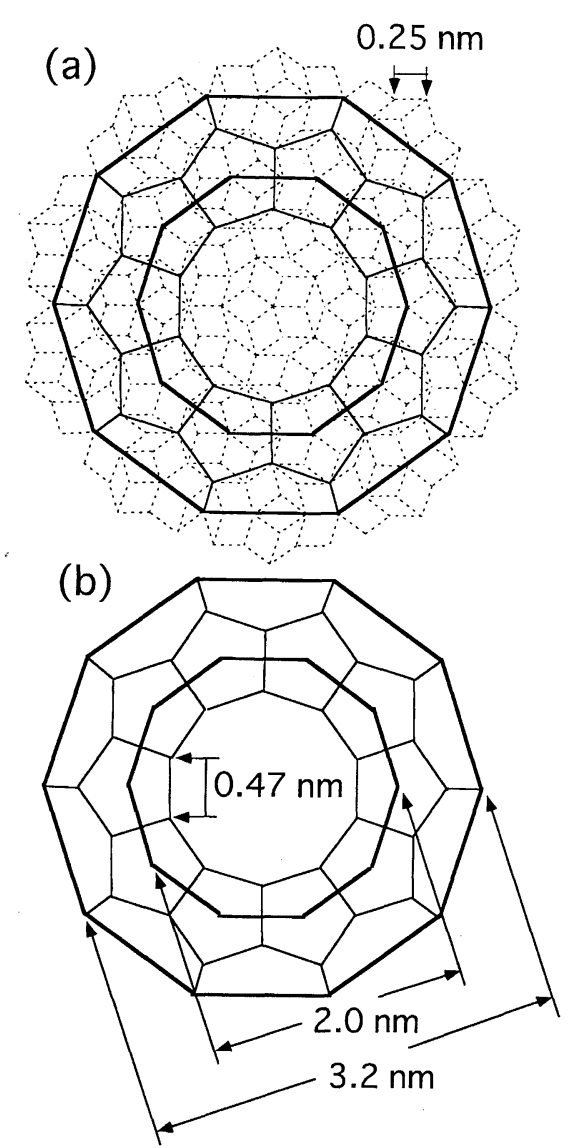

Fig. 8 (a) pentagonal and decagonal frame (thin solid lines), and two decagons with $2.0 \mathrm{~nm}$ and $3.2 \mathrm{~nm}$ in diameter (thick solid lines) in a rhombic Penrose lattice (dotted lines) with a bond length of $0.25 \mathrm{~nm}$. (b) two types of decagons with $2.0 \mathrm{~nm}$ and $3.2 \mathrm{~nm}$ in diameter in the pentagonal and decagonal frame.

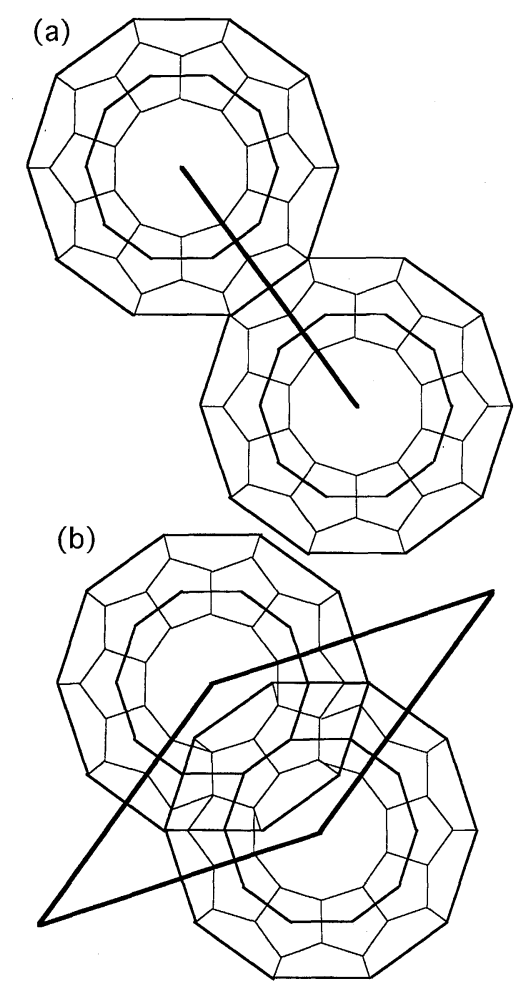

Fig. 9 Linkages of $3.2 \mathrm{~nm}$ clusters by edge-sharing (a) and interpenetrating (b).
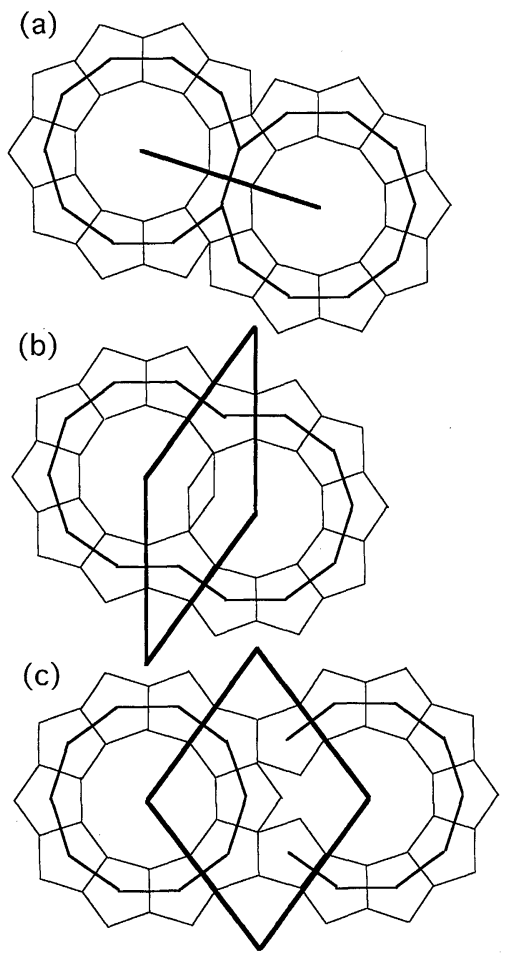

Fig. 10 Edge-sharing linkage of $2.0 \mathrm{~nm}$ clusters (a), and the deformation of the $2.0 \mathrm{~nm}$ clusters in the linkages along short diagonals in thin (b) and fat (c) rhombuses.

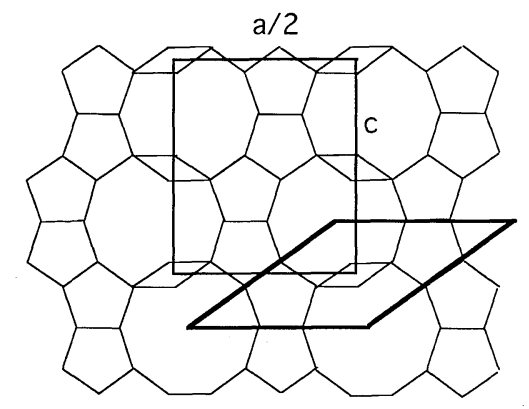

Fig. 11 Pentagonal and decagonal frame of the W-(AlCoNi) structure. A thin rhombus, forming an arrangement of $2.0 \mathrm{~nm}$ clusters, is indicated by thick solid lines. A half of a unit cell is shown by a rectangle.

clearly seen in Figs. 7(b) and (c). As for the W-(AlCoNi) crystalline structure in Fig. 7(d), the $2.0 \mathrm{~nm}$ clusters are connected with each other by the linkages of Figs. 10(a) and (b), and forms a periodic arrangement with the pentagonal and rhombic frames of Fig. 11.

Figure 12 shows HADDF-STEM images of the same samples as those of Fig. 7. Bright contrasts in the HAADF-STEM images correspond to transition-metal atoms, which have large atomic numbers. As a rough approximation, the images of Fig. 12 have reversed contrasts of Fig. 7. In Fig. 12, the $3.2 \mathrm{~nm}$ and $2.0 \mathrm{~nm}$ clusters are indicated by decagons. In Figs. 12(b) and (c), one can notice similar contrast distributions of the $2.0 \mathrm{~nm}$ clusters, which consist of two decagonal circles of bright dots surrounding a pentagonal arrangement of bright dots, and that the clusters are connected with each other by sharing a bright dot in the outer decagonal circle with an interval of $2.0 \mathrm{~nm}$ and by sharing two bright dots in the inner 

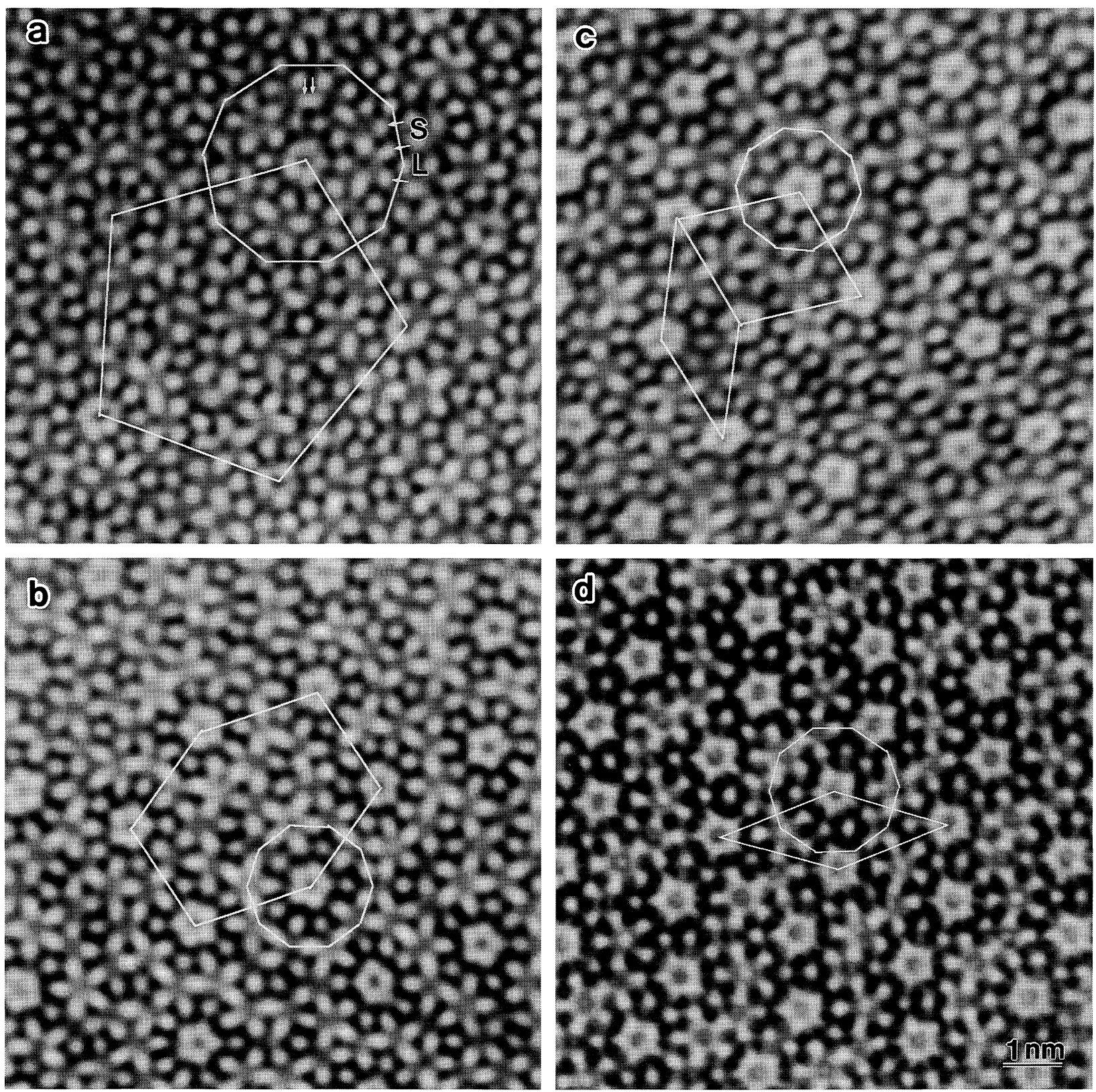

Fig. 12 HAADF-STEM images showing atomic clusters in the Ni-rich basic structure (a), S1-type superstructure (b), type-I superstructure (c) of Al-Co-Ni decagonal quasicrystals, taken with the incident beam parallel to the periodic axis, and in the W-(AlCoNi) crystalline phase (d) taken with the incident beam parallel to the $b$-axis. Decagons show the $3.2 \mathrm{~nm}$ (a) and $2.0 \mathrm{~nm}$ (b-d) clusters. Lattices formed by connecting the centers of the atomic clusters are shown by solid lines.

decagonal circle along a short diagonal of the thin rhombus. This feature can be also seen in the HAADF-STEM image of the W-(AlCoNi) phase (Fig. 12(d)).

On the other hand, the contrast distribution of the $3.2 \mathrm{~nm}$ cluster in Fig. 12(a) consists of fourfold decagonal circles of bright dots surrounding a central contrast. The outer decagonal circles of 20 and 10 bright dots surrounds the inner double decagonal circles, which are similar to those of the $2.0 \mathrm{~nm}$ cluster. From the close examination of the contrast distributions of the clusters in Fig. 12, one can notice that bright dots of the second decagonal circle surrounding central contrasts are slightly elongated along the circumference, as indicated with a pair of small arrows in Fig. 12(a), compared with bright dots in the other decagonal arrangements. Also, one can see that the 20 bright dots of third circles in the $3.2 \mathrm{~nm}$ cluster are arranged with long and short distances, as indicated with $L$ and $S$ in Fig. 12(a). These features will be able to be understood from arrangements of transition-metal atoms, which will be mentioned later, in the $3.2 \mathrm{~nm}$ and $2.0 \mathrm{~nm}$ clusters. Similar HAADF-STEM image contrasts of the $3.2 \mathrm{~nm}$ cluster have been observed in highly-ordered $\mathrm{Al}-\mathrm{Cu}-\mathrm{Rh}^{23)}$ and $\mathrm{Al}-\mathrm{Fe}-\mathrm{Ni}^{24)}$ decagonal quasicrystals.

The pentagonal contrasts at the centers of the $2.0 \mathrm{~nm}$ clusters clearly show two orientations in Fig. 12(b) and the same orientations in Figs. 12(c) and (d). These contrasts can be hardly noticed from the HRTEM images of Fig. 7. Thus, the combination of HAADF-STEM and HRTEM observations can give us more detailed information about the structures of decagonal quasicrystals. 


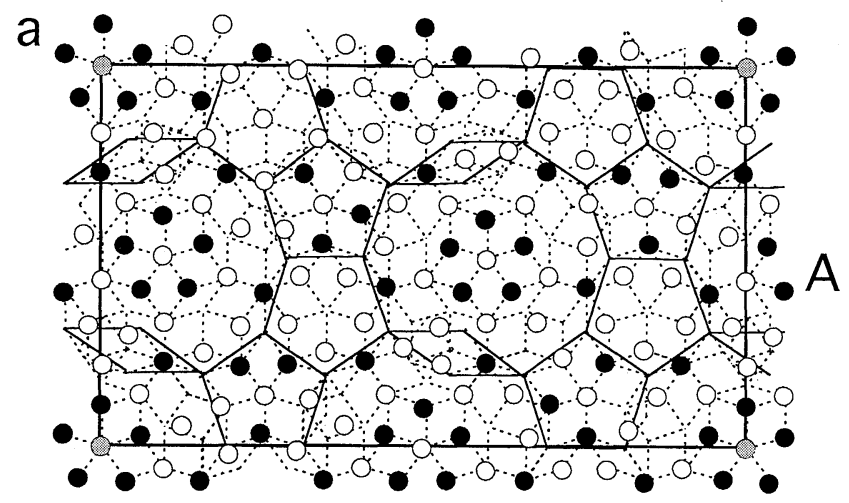

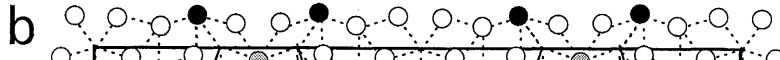

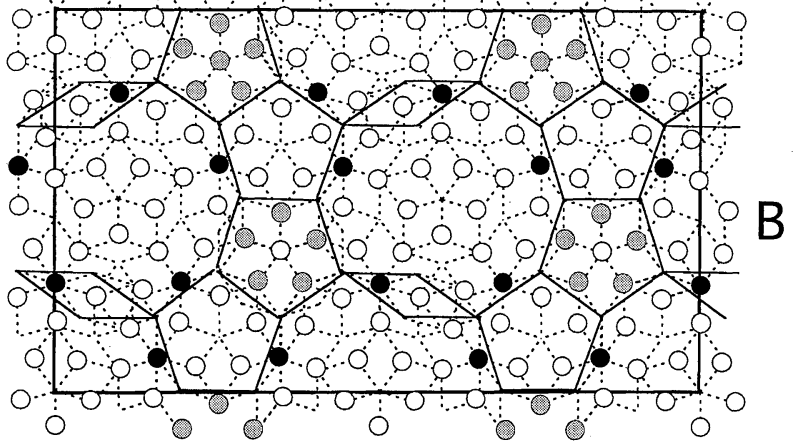

C

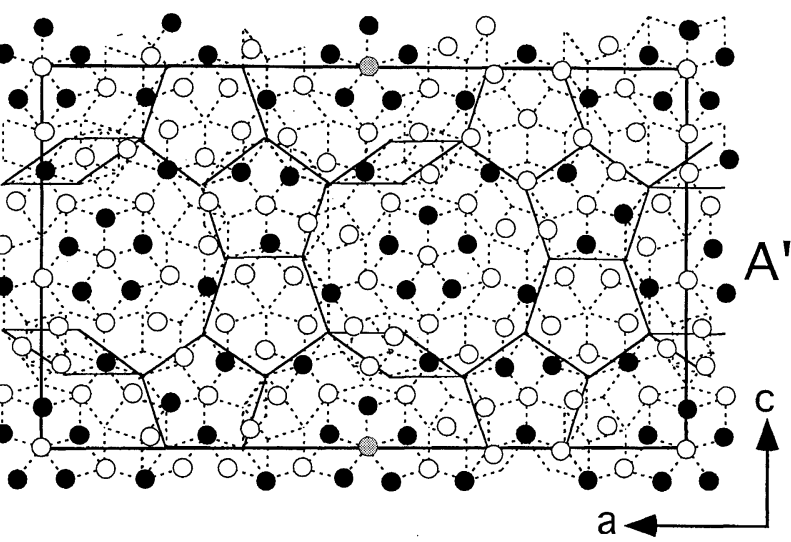

d

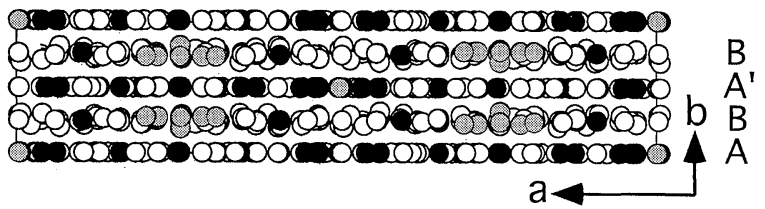

Fig. 13 Structure of the W-(AlCoNi) crystalline phase, which shows atomic arrangements on three layers along the $b$-axis (a-c), and the projection of the structure along the $c$-axis (d). White and black circles correspond to $\mathrm{Al}$ and transition-metal atom positions, respectively, and dark circles to mixed positions of $\mathrm{Al}$ and transition-metal atoms.

\section{Structural Models of Atomic Clusters}

From the HRTEM and HAADF-STEM observations of Al-Co-Ni decagonal quasicrystals and W-(AlCoNi) crystalline phase in Figs. 7 and 12, one can understand that a structural model of the $2.0 \mathrm{~nm}$ cluster forming the structures of all the modulations of the $\mathrm{Al}-\mathrm{Co}-\mathrm{Ni}$ decagonal quasicrystals except for the Ni-rich basic structure can be derived from the structure of the W-(AlCoNi) phase. Also, it should be mentioned here that intensity distributions in the diffraction pat-

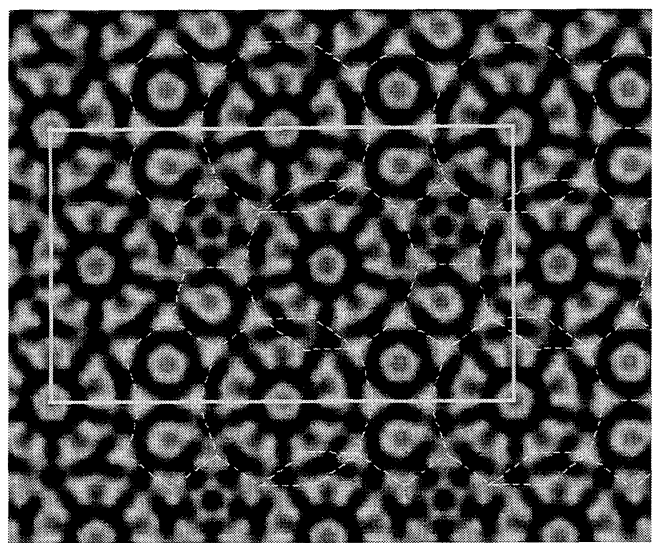

Fig. 14 Simulated HRTEM image calculated from the structural model of Fig. 13 along the $b$-axis, using the multi-slice method with a sample thickness of $4 \mathrm{~nm}$ and a defocus value of $45 \mathrm{~nm}$.

terns of the W-(AlCoNi) crystalline phase closely resemble those of the pentagonal superstructure, although positions of individual diffraction spots are, of course, different in those of the pentagonal superstructure and $\mathrm{W}-(\mathrm{AlCoNi})$ phase. ${ }^{9}{ }^{9}$ Also, an electron diffraction pattern of the $\mathrm{W}$-(AlCoNi) crystalline phase, taken with the incident beam parallel to the $b$-axis, shows pseudo-pentagonal symmetry for some strong spots, so that the $\mathrm{W}-(\mathrm{AlCoNi})$ structure has non-central symmetry, like the pentagonal superstructure. Thus, it can be concluded that the $\mathrm{W}-(\mathrm{AlCoNi})$ is a crystalline approximant closely related to the pentagonal superstructure.

The W-(AlNiCo) phase has a monoclinic structure with lattice parameters $a=3.99 \mathrm{~nm}, b=0.82 \mathrm{~nm}, c=2.36 \mathrm{~nm}$ and $\beta=90^{\circ}$, and the space group of $\mathrm{Cm}$ (No. 8). ${ }^{9)}$ Figure 13 shows an atomic arrangement of the $\mathrm{W}-(\mathrm{AlCoNi})$ phase, which was determined by the single crystal X-ray diffraction, in the pentagonal and decagonal frame, and rhombic Penrose lattice. The structure of the $\mathrm{W}-(\mathrm{AlCoNi})$ phase can be described by four layers along the $b$-axis. The layer of $y=1 / 2$ is a mirror plane, so atomic arrangements in the $y=1 / 4$ and $3 / 4$ layers have a mirror symmetry. On the other hand, the atomic arrangement of $y=1 / 2$ corresponds to that of $y=0$ shifted with $x=1 / 2$ along the $a$-axis. Therefore, the structure of the $\mathrm{W}$-(AlCoNi) phase has a stacking of $A B A^{\prime} B$ along the $b$-axis. All atoms on the $A$ and $A^{\prime}$ layers are located on $y=0$ and $1 / 2$ planes, respectively, whereas atoms on the $B$ layer are distributed in the range of $y=1 / 4 \pm 0.06$, as can be seen in Fig. 13(d), in which the projection of the structure along the $c$-axis is shown. However, it should be noted here that atoms distributed in the wide range are $\mathrm{Al}$ atom and mixed atom positions, and transition-metal atom positions are localized in the narrow range of $y=1 / 4 \pm 0.002$.

In Figs. 13(a)-(c), one can see that transition-metal atoms are completely placed at lattice points of the rhombic Penrose lattice and form pentagonal lattices of bond lengths of $0.49 \mathrm{~nm}$ in $A$ and $0.77 \mathrm{~nm}$ in $B$ layer. On the other hand, $\mathrm{Al}$ atoms are located at Penrose lattice points in the $B$ layer, but some of $\mathrm{Al}$ atoms in the $A$ layer are remarkably shifted from the lattice points. This shift and also the distribution of atoms in wide regions around $y=1 / 4$ and $3 / 4$ planes in the $B$ layer are caused by triangle arrangements of transition-metal atoms with slightly short atomic radiuses in the pentagonal 


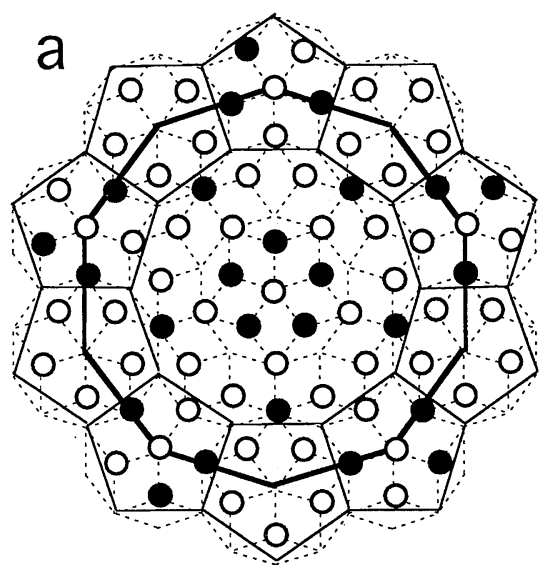

A

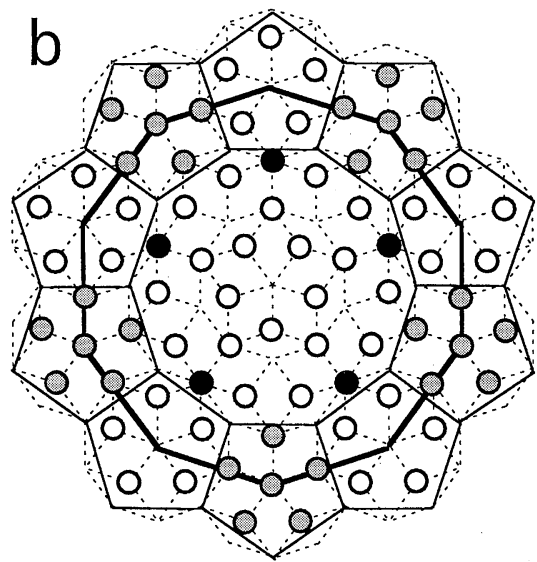

B

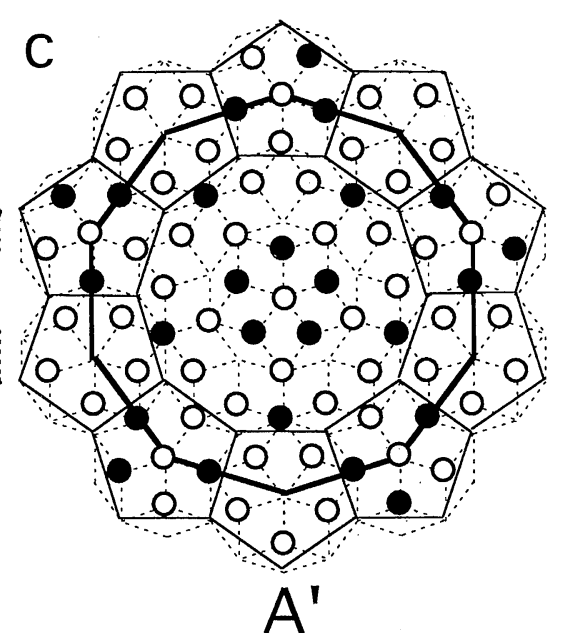

Fig. 15 Ideal structure model of the $2.0 \mathrm{~nm}$ cluster with $0.8 \mathrm{~nm}$ periodicity, which is showed by atomic arrangements on three layers. The structure of the clusters have a sequence of $A B A^{\prime} B$ along the columnar axis. White and black circles correspond to $\mathrm{Al}$ and transition-metal atom positions, respectively, and dark circles to mixed positions of $\mathrm{Al}$ and transition-metal atoms.

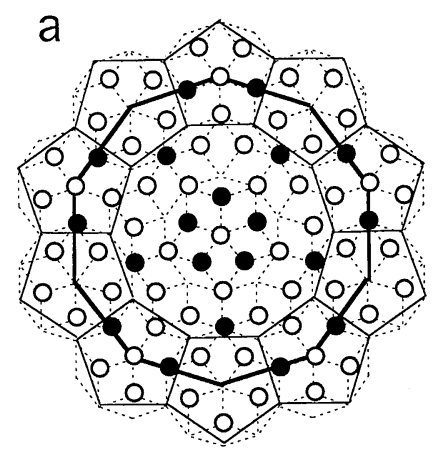

A

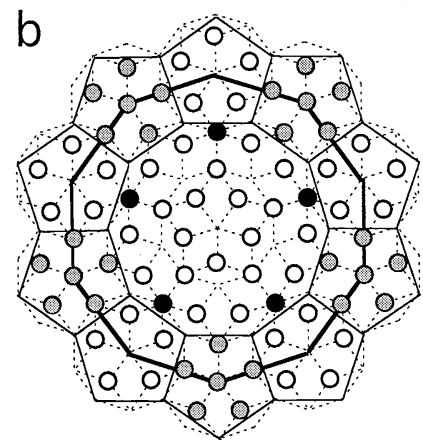

B
Fig. 16 Ideal structure model of the $2.0 \mathrm{~nm}$ cluster with $0.4 \mathrm{~nm}$ periodicity, which is showed by atomic arrangements on two layers. The structure of the cluster has a sequence of $A B$ along the columnar axis. White and black circles correspond to $\mathrm{Al}$ and transition-metal atom positions, respectively, and dark circles to mixed positions of $\mathrm{Al}$ and transition-metal atoms.

frames of the $A$ and $A^{\prime}$ layers. However, an HRTEM image of the $\mathrm{W}-(\mathrm{AlCoNi})$ phase, calculated from the model of Fig. 13 along the $b$-axis replicates well the contrast of Fig. 7(d), particularly ring contrasts and wheel-like contrasts, as shown in Fig. 14.

From the W-(AlCoNi) structure, it is worthwhile to derive structure models of the atom clusters. Figure 15 shows an ideal model of the $2.0 \mathrm{~nm}$ cluster with $0.8 \mathrm{~nm}$ periodicity, without taking account of the atomic shifts from the Penrose lattice points. The structure of this cluster has a stacking of $A B A^{\prime} B$ along the columnar axis. It should be noted here that atoms in the $A$ and $A^{\prime}$ layers are placed on the planes of $z=0$ and $1 / 2$ ( $z$ is a coordinate along the columnar axis of the cluster), but most of $\mathrm{Al}$ atom and mixed atom positions in the $B$ layer are distributed in some regions around $z=0$ and $1 / 2$. In the model, one can notice that the $0.8 \mathrm{~nm}$ periodicity of the cluster results from orientations of the rectangle arrangements of transition-metal atoms in the pentagonal frames. Therefore, the $2.0 \mathrm{~nm}$ cluster with $0.4 \mathrm{~nm}$ periodicity, which are found in the modulations in the Ni-rich side, can be presumed to have a structure of Fig. 16. In this model, each of the tri-

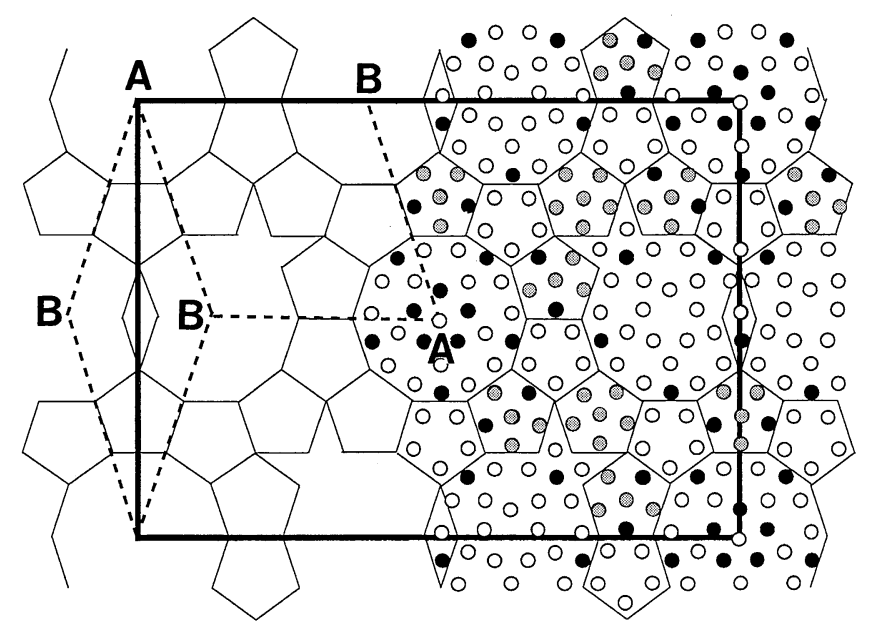

Fig. 17 Atomic arrangement (on one layer) of a crystalline approximant found in an $\mathrm{Al}_{71.5} \mathrm{Co}_{16} \mathrm{Ni}_{12.5}$ alloy, obtained by placing the structural model of Fig. 16 in the pentagonal and decagonal frame of an HRTEM image observed from this crystalline approximant. The atomic arrangement is shown in a half part of a unit cell. Dotted lines show thin and fat rhombuses. One can see three linkages of $2.0 \mathrm{~nm}$ clusters, shown in Fig. 10, in this structure. White and black circles correspond to $\mathrm{Al}$ and transition-metal atom positions, respectively, and dark circles to mixed positions of $\mathrm{Al}$ and transition-metal atoms. $A$ and $B$ show the clusters with two different orientations of pentagonal symmetry.

angle arrangements of transition-metal atoms in pentagonal frames of Fig. 15 is replaced by a pair of two transition-metal atoms. Consequently, this cluster has a stacking of $A B$ along the columnar axis.

The structures of most of the modulations of $\mathrm{Al}-\mathrm{Co}-\mathrm{Ni}$ decagonal quasicrystals and their crystalline approximants can be constructed by placing the structural model of the $2.0 \mathrm{~nm}$ cluster of Fig. 15 or Fig. 16 in the pentagonal and decagonal frames of observed HRTEM images, such as Figs. 7(b) and (c). Figure 17 shows a structure model of a crystalline approximant found in an $\mathrm{Al}_{71.5} \mathrm{Co}_{16} \mathrm{Ni}_{12.5}$ alloy annealed at $900^{\circ} \mathrm{C}$ for $120 \mathrm{~h}$. The crystalline phase, which is closely related to the type-I Al-Co-Ni decagonal quasicrystal, has an orthorhombic structure with lattice param- 

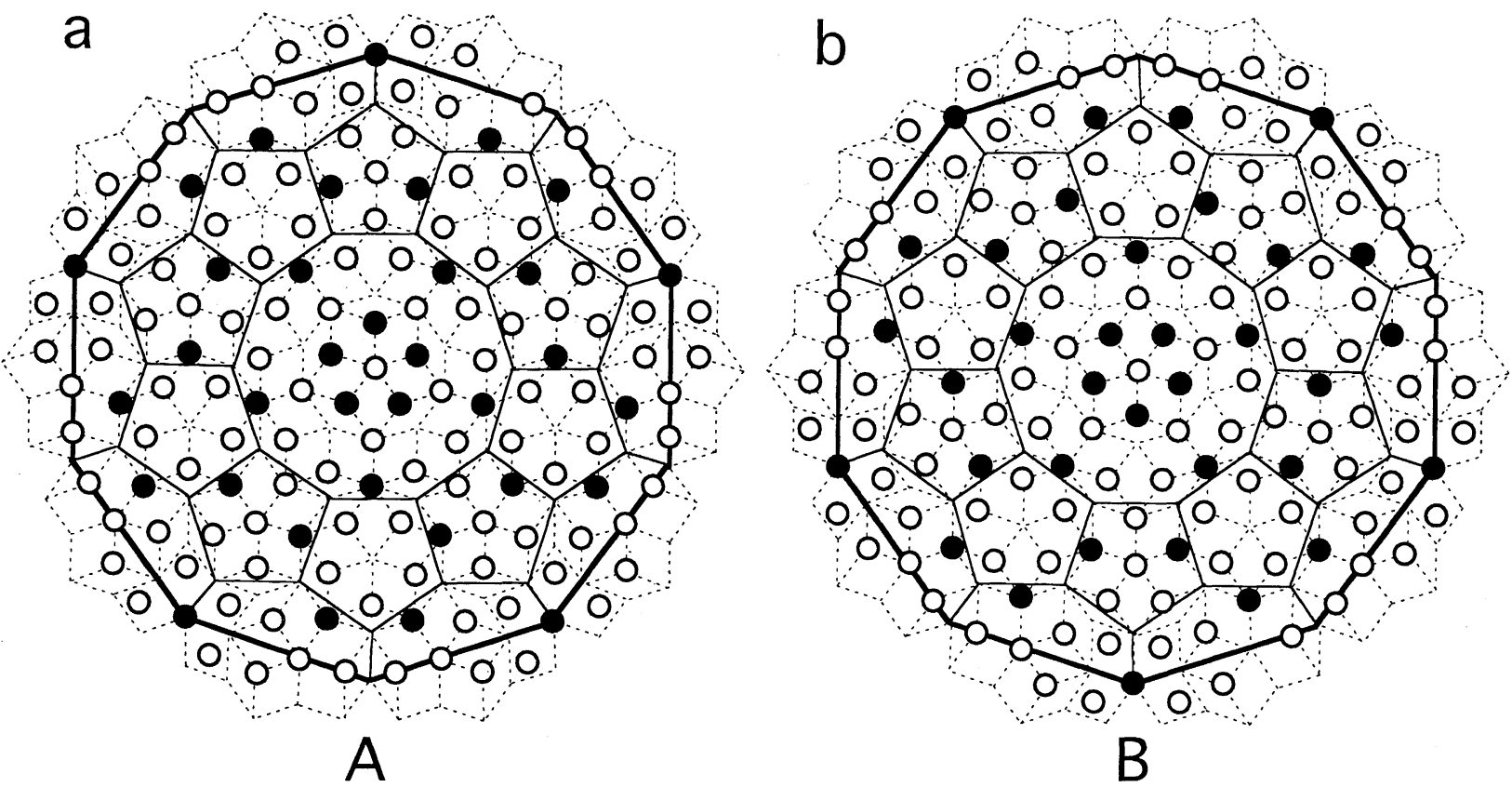

Fig. 18 Ideal structural model of the $3.2 \mathrm{~nm}$ decagonal columnar cluster with a $0.4 \mathrm{~nm}$ period along the columnar axis. The structure of the cluster has a sequence of $A B$ along the columnar axis. White and black circles correspond to Al and transition-metal atom positions, respectively.

Table 2 Structural characteristics of Al-Co-Ni decagonal quasicrystals and crystalline approximants.

\begin{tabular}{lll}
\hline & \multicolumn{1}{c}{ Tiling } & Atomic cluster \\
\hline Ni-rich basic structure & Pentagonal & $3.2 \mathrm{~nm}$ cluster \\
S1-type superstructure & Pentagonal & $2.0 \mathrm{~nm}$ cluster, two orientations \\
Type-I superstructure & Rhombic & $2.0 \mathrm{~nm}$ cluster, two orientations \\
Type-II superstructure & Pentagonal + rhombic & $2.0 \mathrm{~nm}$ cluster, two orientations \\
Crystalline approximant & Rhombic & $2.0 \mathrm{~nm}$ cluster, two orientations \\
Co-rich basic structure & Pentagonal & $2.0 \mathrm{~nm}$ cluster, one orientation \\
One-dimensional quasicrystal & Pentagonal + rhombic & $2.0 \mathrm{~nm}$ cluster, two orientations \\
W-(AlCoNi) approximant & Rhombic & $2.0 \mathrm{~nm}$ cluster, one orientation \\
Pentagonal superstructure & Rhombic & $2.0 \mathrm{~nm}$ cluster, one orientation \\
\hline
\end{tabular}

eters $a=5.2 \mathrm{~nm}, b=0.4 \mathrm{~nm}$ and $c=3.7 \mathrm{~nm} .{ }^{13)}$ From HAADF-STEM and HRTEM observations of the crystalline phase, its structure can be characterized by a periodic arrangement of the $2.0 \mathrm{~nm}$ clusters of two orientations in a tiling of thin and fat rhombuses, as indicated by dotted lines in Fig. 17, and described by a pentagonal and decagonal frame shown in Fig. 17. ${ }^{13)}$ By assuming that these two types of clusters, $A$ and $B$, are rotated at 180 degrees and shifted at $c / 2$ ( $c$ is a period along the columnar axis in Fig. 16), an atomic arrangement of one layer in the crystalline phase can be derived easily from the structural model of Fig. 16, as shown in Fig. 17. Thus, this atomic arrangement can be obtained directly by placing the structural model of Fig. 16 in the pentagonal and decagonal frame obtained from an HRTEM image of this crystalline approximant, although there is the ambiguity for transitionmetal and mixed positions.

As mentioned in Figs. 2 and 3, the diffuse scattering showing $0.8 \mathrm{~nm}$ periodicity is always observed in diffraction patterns of all the modulations besides the Ni-rich basic structure, and its intensity is gradually enhanced with increasing Co content. The diffuse scattering with peaks at definite positions associated with the golden ratio can be considered to be caused by the appearance of the clusters with $0.8 \mathrm{~nm}$ periodicity (Fig. 15) in an arrangement of the clusters of $0.4 \mathrm{~nm}$ periodicity (Fig. 16), or by the appearance of atomic arrangements like Fig. 15 in the structure of Fig. 16.

From the structural model of the $2.0 \mathrm{~nm}$ cluster with $0.4 \mathrm{~nm}$ periodicity, an ideal model of the $3.2 \mathrm{~nm}$ cluster in the Ni-rich basic structure can be derived, as shown in Fig. 18. The diffraction patterns (Figs. 2(a) and 3(a)) of the Ni-rich basic structure show that the $3.2 \mathrm{~nm}$ cluster has the $c$-glide ( $c$ is the periodic axis) plane or a $10_{5}$ screw axis along the $c$-axis. Also, an arrangement of transition-metal atoms can be estimated from the HAADF-STEM image of Fig. 12(a). The structure of Fig. 18 is proposed as a positive model, which is derived from the HRTEM and HAADF-STEM images of the Ni-rich basic structure, and also by the speculation from the structures of the $2.0 \mathrm{~nm}$ clusters.

The structure models of the $2.0 \mathrm{~nm}$ and $3.2 \mathrm{~nm}$ clusters (Figs. 15, 16 and 18) can be easily inferred to give contrast distributions of the atom clusters in the observed HRTEM images (Fig. 7), from the structure and simulated image of the $\mathrm{W}-(\mathrm{AlCoNi})$ phase (Figs. 13 and 14). Also, it is easily understood that bright dots in HAADF-STEM images of the 
$2.0 \mathrm{~nm}$ and $3.2 \mathrm{~nm}$ clusters (Fig. 12) correspond to transitionmetal atoms in the projections of the $2.0 \mathrm{~nm}$ and $3.2 \mathrm{~nm}$ clusters (Figs. 15, 16 and 18) along the columnar axes. The bright dot elongated along the circumference in the second decagonal circle surrounding the central contrast corresponds to two transition-metal atoms separated with an interval of $0.15 \mathrm{~nm}$, and the bright dots arranged with two intervals of $S$ and $L$ (Fig. 12(a)) in the third decagonal circle in the $3.2 \mathrm{~nm}$ cluster to transition-metal atoms arranged with $0.40 \mathrm{~nm}$ and $0.47 \mathrm{~nm}$ intervals.

Finally, it should be noted that the ideal structural models of $2.0 \mathrm{~nm}$ and $3.2 \mathrm{~nm}$ clusters, proposed in this paper, closely resemble those estimated from only HRTEM and HAADF-STEM observations in the previous papers. ${ }^{9-19)}$ However, it should be mentioned here that the present Ni-rich basic structure formed by an aperiodic arrangement of the $3.2 \mathrm{~nm}$ clusters is quite different from some structural models previously proposed by only HRTEM or HAADF-STEM observation. ${ }^{27-30)}$

\section{Summary}

Structural characteristics of seven modulations of Al-Co-Ni decagonal quasicrystals and two crystalline approximants, which are formed in and around an $\mathrm{Al}-\mathrm{Co}-\mathrm{Ni}$ decagonal phase with a wide compositional range, are systematically discussed. Except for the Ni-rich basic structure formed with columnar atom clusters with decagonal sections of $3.2 \mathrm{~nm}$ in diameter, the structures of the other modulations and crystalline approximants are formed by columnar atom clusters with decagonal sections of $2.0 \mathrm{~nm}$ in diameter. The $2.0 \mathrm{~nm}$ clusters have pentagonal symmetry, so that tilings of the clusters and ordered arrangements of the clusters with two orientations of pentagonal symmetry produce many types of modulations of decagonal quasicrystals with different intensity distributions of diffraction patterns and crystalline approximants. In Table 2, the structural characteristics of the modulations of decagonal quasicrystals and crystalline approximants are summarized. From Table 2, one can notice possibility of the appearance of various types of modulations of decagonal quasicrystals, which result from the combination of rhombic and pentagonal tilings, and ordered arrangements of the atomic clusters with two orientations of pentagonal symmetry in the tilings.

\section{REFERENCES}

1) A. P. Tsai, A. Inoue and T. Masumoto: Mater. Trans., JIM 30 (1989) 150-154.

2) K. Hiraga, F. J. Lincoln and W. Sun: Mater. Trans., JIM 32 (1991) 308-314.

3) K. Edagawa, M. Ichihara, K. Suzuki and S. Takeuchi: Philos. Mag. Lett. 66 (1992) 19-25.

4) A. P. Tsai, A. Fujiwara, A. Inoue and T. Masumoto: Philos. Mag. Lett. 74 (1996) 233-240.

5) S. Ritsch, C. Beeli, H.-U. Nissen, T. Godecke, M. Scheffer and R. Lück: Philos. Mag. Lett. 78 (1998) 67-75.

6) B. Grushko, D. Holland-Moritz, R. Wittmann and G. Wilde: J. Alloys and Comp. 280 (1998) 215-230.

7) K. Hiraga, W. Sun and A. Yamamoto: Mater. Trans., JIM 35 (1994) 657-662.

8) A. P. Tsai, A. Inoue and T. Masumoto: Philo. Mag. Lett. 71 (1995) 161-167.

9) K. Hiraga, T. Ohsuna and S. Nishimura: J. Alloy and Comp. 325 (2001) $145-150$.

10) K. Sugiyama, S. Nishimura and K. Hiraga: to be published in J. Alloy and Comp.

11) K. Hiraga, T. Ohsuna and S. Nishimura: Philos. Mag. Lett. 80 (2000) 653-659.

12) K. Hiraga, T. Ohsuna, S. Nishimura and M. Kawasaki: Philos. Mag. Lett. 81 (2001) 109-115.

13) K. Hiraga, T. Ohsuna and S. Nishimura: Mater. Trans. 42 (2001) 1081-1084.

14) K. Hiraga, T. Ohsuna and S. Nishimura: Philos. Mag. Lett. 81 (2001) 123-127.

15) K. Hiraga and T. Ohsuna: Mater. Trans. 42 (2001) 509-513.

16) K. Hiraga, W. Sun and T. Ohsuna: Mater. Trans. 42 (2001) 1146-1148.

17) K. Hiraga, T. Ohsuna and S. Nishimura: Mater. Trans. 42 (2001) 18301833.

18) K. Hiraga, T. Ohsuna, K. Yubuta and S. Nishimura: Mater. Trans. 42 (2001) 897-900.

19) K. Hiraga: Advances in IMAGING and ELECTRON PHYSICS (Academic Press, 1998, ed. P. W. Hawkes) 101, pp. 37-98.

20) K. Niizeki: J. Phys. Soc. Jpn. 63 (1994) 4035-4043.

21) T. Ohsuna, W. Sun and K. Hiraga: Philos. Mag. Lett. 80 (2000) 577-583.

22) K. Hiraga, T. Ohsuna and W. Sun: Mater. Sci. and Eng. 312 (2001) 1-8.

23) K. Hiraga, T. Ohsuna and K.-T. Park: Philos. Mag. Lett. 81 (2001) 117-122.

24) K. Hiraga and T. Ohsuna: Mater. Trans. 42 (2001) 894-896.

25) K. Sugiyama, N. Kaji, K. Hiraga and T. Ishimasa: Z. Kristallogr. 213 (1998) 90-95.

26) K. Sugiyama, T. Kato, T. Ogawa, K. Hiraga and K. Saito: J. Alloys and Comp. 299 (2000) 169-174.

27) K. Saitoh, K. Tsuda, M. Tanaka, K. Kaneko and A. P. Tsai: Jpn. J. Appl. Phys. 36 (1997) L1400-L1402.

28) Y. Yan, S. J. Pennycook and A. P. Tsai: Phys. Rev. Lett. 81 (1998) 5145-5148.

29) E. Abe, K. Saitoh, M. Tanaka, A. P. Tsai, P. J. Steinhardt and H.-C. Jeong: Phys. Rev. Lett. 84 (2000) 4609-4612.

30) P. J. Steinhardt, H.-C. Jeong, K. Saitoh, M. Tanaka, E. Abe and A. P. Tsai: Nature, 396, November (1998) 55-57. 